\title{
Variables intermedias de la fecundidad adolescente en Chile: Evolución, desigualdad e implicancias de políticas
}

\author{
JORGE RODRÍGUEZ ${ }^{(1)}$
}

\section{RESUMEN}

Haciendo uso de las escasas y limitadas encuestas poblacionales disponibles en Chile se describe la evolución de la iniciación sexual y del uso de anticonceptivos, tanto en términos de magnitud como de desigualdad social y de género. La iniciación sexual se adelanta, deviene mayoritariamente prenupcial y se reducen las diferencias según género. El uso de anticonceptivos en la primera relación sexual aumenta, aunque sigue siendo inferior al de los países desarrollados 20 años atrás. La desigualdad social en el uso de anticonceptivos en la primera unión es más pronunciada que la de la iniciación sexual. Esto refuerza la prioridad de universalizar el acceso a anticonceptivos desde la primera relación sexual, para que cada adolescente pueda decidir libre, informada y conscientemente sobre su uso, sin restricciones adicionales a las establecidas legalmente.

Palabras clave: fecundidad adolescente, iniciación sexual, uso de anticonceptivos, desigualdad social.

\section{ABSTRACT}

INTERMEDIATE VARIABLES IN THE ADOLESCENT FERTILITY RATE IN CHILE: EVOLUTION, INEQUALITY AND POLICY IMPLICATIONS

Making use of the scarce and limited population surveys available in Chile, the evolution of age at first intercourse and the use of contraceptives is described, both in terms of magnitude and social and gender inequality. Age at first intercourse has become younger, primarily premarital, and gender differences have narrowed. The use of contraceptives at first sexual intercourse has increased, although it continues to be lower than in developed countries 20 years ago. Social inequality in the use of contraceptives at first intercourse is more pronounced than inequality in first intercourse itself. This underlines the priority of universalizing access to contraceptives, starting with the first sexual encounter, so that each adolescent can make their decision about contraceptives freely, consciously and well informed, without additional restrictions to those that are legally established.

Keywords: adolescent fertility, sexual initiation, use of contraceptives, social inequality.

(1) Centro Latinoamericano y Caribeño de Demografía (CELADE). División de Población de la CEPAL. Avda Dag Hammarskjold 3477. Vitacura. Santiago. Chile. jorge.rodriguez@cepal.org 


\section{INTRODUCCIÓN}

La evidencia mundial disponible sugiere que la modernidad -proceso cultural basado en la individuación, la secularización y la reflexividadtransforma la intimidad, la sexualidad y las relaciones familiares en un sentido que deja a los y las adolescentes más espacio para decisiones autónomas, es decir, con una decreciente injerencia de padres u otros referentes de autoridad adultas. En la experiencia de los países desarrollados, en particular los occidentales, esto se ha expresado en tres tendencias: a) una iniciación sexual más temprana; b) una iniciación sexual previa al matrimonio o a la cohabitación; c) una igualación de la edad de iniciación sexual entre hombres y mujeres ${ }^{1}$.

En su investigación de alcance mundial, Bozón plantea la existencia de tres modelos de "acceso a la sexualidad", en función del diferencial entre hombres y mujeres en cada uno. Y uno de esos modelos es el latinoamericano, donde el control social (sobre todo el ejercido por la familia) promueve la protección de la virginidad hasta el matrimonio o unión para las mujeres, pero incentiva la iniciación temprana y prematrimonial entre los hombres ${ }^{2}$. Por lo anterior, el adelantamiento de la iniciación sexual y la expansión de la actividad prematrimonial entre las latinoamericanas que registran las encuestas especializadas ${ }^{3}$, se ha dado en un contexto social más hostil o al menos más ambivalente frente a estos cambios. Y lo anterior parece tener efectos colaterales, sobre todo en materia de control de los efectos reproductivos de este nuevo patrón de iniciación sexual. En particular, los niveles de fecundidad adolescente en América Latina y en Chile son mucho más elevados de lo que cabría esperar a la luz de sus índices de fecundidad total y de desarrollo humano ${ }^{4}$.

La fecundidad adolescente en América Latina y en Chile también es muy desigual en términos socioeconómicos ${ }^{5}$. La mayor parte de los estudios $^{\mathrm{a}}$ sobre el tema ha mostrado que lo anterior se explica por una doble desigualdad de sus variables intermedias ${ }^{\mathrm{b}}$ : i) menor nivel socioeconómico se asocia a iniciaciones sexuales más tempranas; ii) menor nivel socioeconómico se asocia a menores índices de uso de anticonceptivos. Es probable que el acceso al aborto y, sobre todo, las condiciones en que se practica, también sea diferenciado entre grupos socioeconómicos, pero hay muy poca evidencia disponible sobre el tema en Chile.

Este trabajo procura describir la tendencia global y según grupos socioeconómicos de las dos variables intermedias principales de la fecundidad adolescente, a saber: la actividad sexual durante la adolescencia (en particular la edad de iniciación sexual), y el uso de anticonceptivos (en particular, el uso en la primera relación sexual). Este ejercicio permitirá tener una aproximación, primera y gruesa, a la importancia relativa de cada una de estas variables sobre la evolución de la intensidad y la desigualdad sociales de la fecundidad adolescente en el país. Se trata de un asunto muy relevante en materia de políticas, por cuanto la maternidad temprana tiene adversidades bien documentadas, tanto para los progenitores como para los niños/as .

\section{MATERIAL Y MÉTODO}

Las fuentes de información para el análisis de las variables intermedias de la fecundidad adolescente en Chile son escasas y limitadas. En este trabajo se usan las tres encuestas poblacionales con representatividad nacional disponibles: el Estudio Nacional de Comportamiento Sexual, 19987 (Comisión Nacional del SIDA-CONASIDA, 2000); las cinco encuestas nacionales de juventud (ENJUVE) levantadas por el Instituto Nacional

(a) Para un listado detallado de los estudios sobre el tema ver Dides y Benavente. 2008. Tabla 4.

(b) En el caso de la fecundidad adolescente, éstos serían: la menarquia y los niveles de esterilidad natural, la iniciación y actividad sexual, la iniciación de la unión, el uso de anticonceptivos y el aborto (Rodríguez, 2009; Bongaarts, 1978). 
de la Juventud (INJUV) entre 1994-2006 ${ }^{\mathrm{c}}$ y las dos encuestas nacionales de calidad de vida (ENCV), levantadas por el Ministerio de Salud (Minsal) en 2000 y $2006^{\mathrm{d}}$.

La metodología es cuantitativa y se basa principalmente en el procesamiento de las bases de microdatos de algunas de las encuestas mencionadas. Se seleccionaron indicadores técnicamente pertinentes. Con estos indicadores se elaboraron series de tiempo, en general como dos o tres momentos a lo más, o valores por cohortes para examinar tendencias. Se efectuaron cruces de variables para examinar asociación. Finalmente, se realizaron análisis multivariados mediante regresiones logísticas para controlar factores exógenos. Todos los procesamientos se realizaron con las bases de datos debidamente ponderadas. La información detallada sobre tamaño muestral, tasas de respuestas y errores muestrales está disponible en los informes oficiales de las encuestas usadas.

Con la información existente en Chile es imposible aplicar modelos microdemográficos para estimar el efecto específico de cada variable intermedia; intento que incluso contando con fuentes especializadas es complejo ${ }^{8}$. En el caso del uso de anticonceptivos, por ejemplo, esta estimación requiere de fuentes que permitan evaluar el riesgo experimentado en cada relación sexual en función de las condiciones de fecundabilidad y la eficiencia del medio usado en cada una de ellas. El uso de modelos

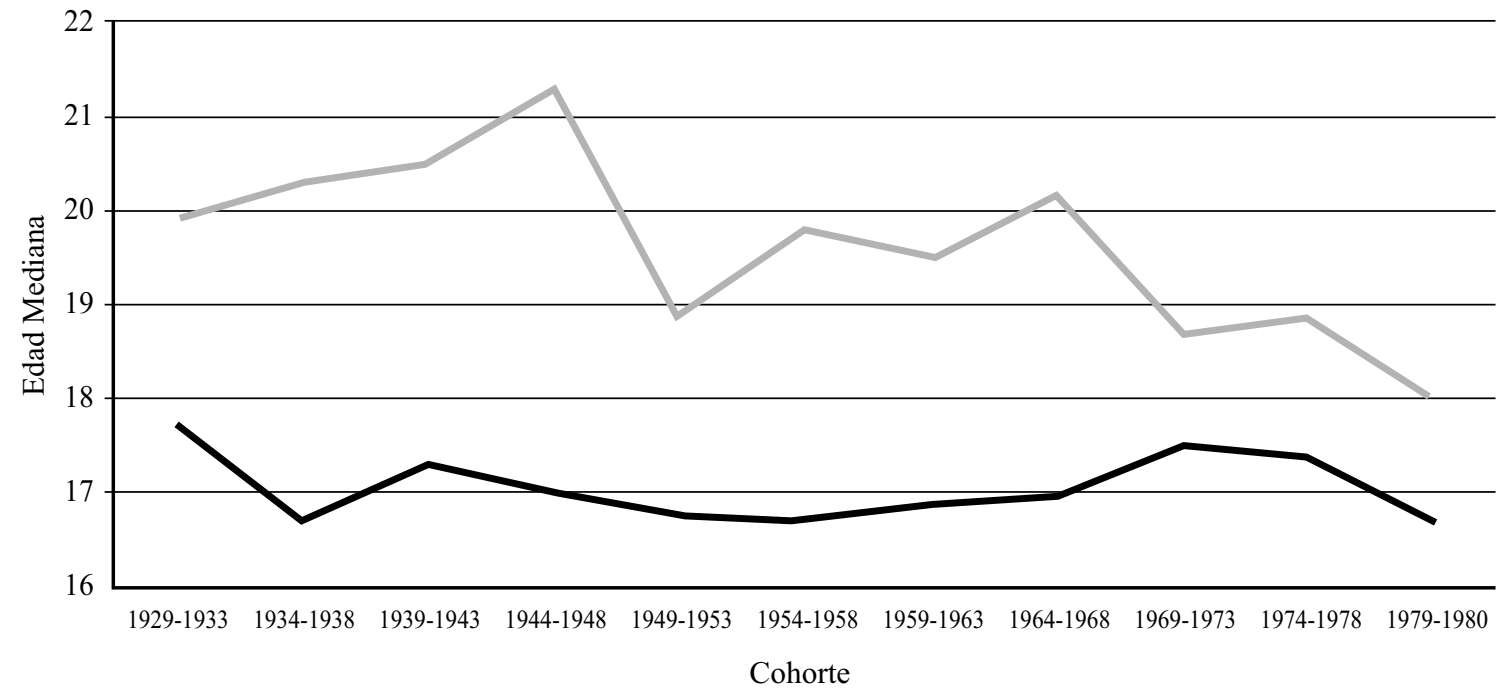

Mujeres $\quad$ Hombres

Fuente: CONASIDA, 2000, p. 31.

Figura 1. Chile. Edad mediana de iniciación sexual, por sexo y cohorte.

(c) Se dispuso de la base de datos de las encuestas de 2003 y 2006 y para ciertos procesamientos en línea se usó INJUMAP (http://celade.cepal.org/redatam/paises/chl/injumapIII). Los informes de las cinco encuestas están disponibles en: http://www.injuv.gob.cl/modules.php?name=Content\&pa=showpage \&pid=4).

(d) En el caso de la ENCV 2000 no se dispuso de la base de datos, pero sí del Informe del módulo de planificación familiar (http://epi.minsal.cl/epi/html/sdesalud/cdevid/infplanfam.pdf). Este módulo tenía una limitación importante (no consultó sobre iniciación sexual ) por lo que virtualmente se excluyó del análisis. En el caso de la ENCV 2006 sí se dispuso de la base de datos en formato SPSS. El informe de la ENCV 2006 está disponible en: http://epi.minsal.cl/epi/html/sdesalud/calidaddevida2006/Informe\%20Final\%20Encuesta\%20de\%20Calidad\%20d e\%20Vida\%20y\%20Salud\%202006.pdf 
macrodemográficos ${ }^{9}$, es una opción a explorar en futuros trabajos, aunque en el caso de la fecundidad adolescente algunos de los indicadores tradicionales que requieren como insumo presentan debilidades. Es el caso, por ejemplo de la prevalencia anticonceptiva ("uso actual"). En efecto, la evidencia indica que luego de tener un primer hijo, las barreras sociales e institucionales para el acceso a la anticoncepción por parte de adolescentes se reducen ${ }^{10}$. Por ello, una fracción de las adolescente usuarias son madres y, por ende, su uso actual sirve para evitar un segundo hijo más no la maternidad adolescente.

\section{RESULTADOS}

\section{Iniciación sexual}

La iniciación sexual de las mujeres se ha adelantado en las últimas décadas, mientras que en el caso de los hombres se ha mantenido más bien estable, o incluso ha aumentado. La edad mediana de la primera relación sexual bajó desde 20 años para la cohorte nacida entre 1929 y 1933 a 18 años en la cohorte nacida en 19791980 (Figura 1). Usando el indicador proporción de iniciadas sexualmente antes de los 18 años, la ENCV 2006 detecta un aumento de esta proporción desde el 30\% para la cohorte de nacidas antes de la década de 1950 a un $40 \%$ entre la cohorte de nacidas en el período 19841988 (Figura 2).

La serie de tiempo que permite construir las 5 ENJUVE muestra una novedad, pues entre 2000 y 2006 este proceso de adelantamiento de la iniciación sexual se habría detenido (Figura 3). Se trata de un hallazgo hasta ahora poco difundido y discutido, y que genera implicancias de política y desafíos teóricos. Lamentablemente, la información está disponible para el grupo adolescente consolidado (ambos sexos), por lo que este hallazgo podría no ser representativo de la tendencia de las mujeres, que son, según las Figuras 1 y 2, las que están adelantando la iniciación sexual (a diferencia de los hombres donde la tendencia es menos clara). Por esto último, este hallazgo debe ser tomado con cautela y ciertamente requiere más investigación.

Por otra parte, las cifras de las Figuras $1 \mathrm{y}$ 2 revelan que la brecha de la iniciación según genero aún existe -y en el sentido esperado, es decir los hombres declaran una iniciación más temprana-, aun cuando su tendencia es al estrechamiento.

Respecto del sexo prematrimonial, las dos fuentes usadas en las Figuras 1 y 2 carecen de

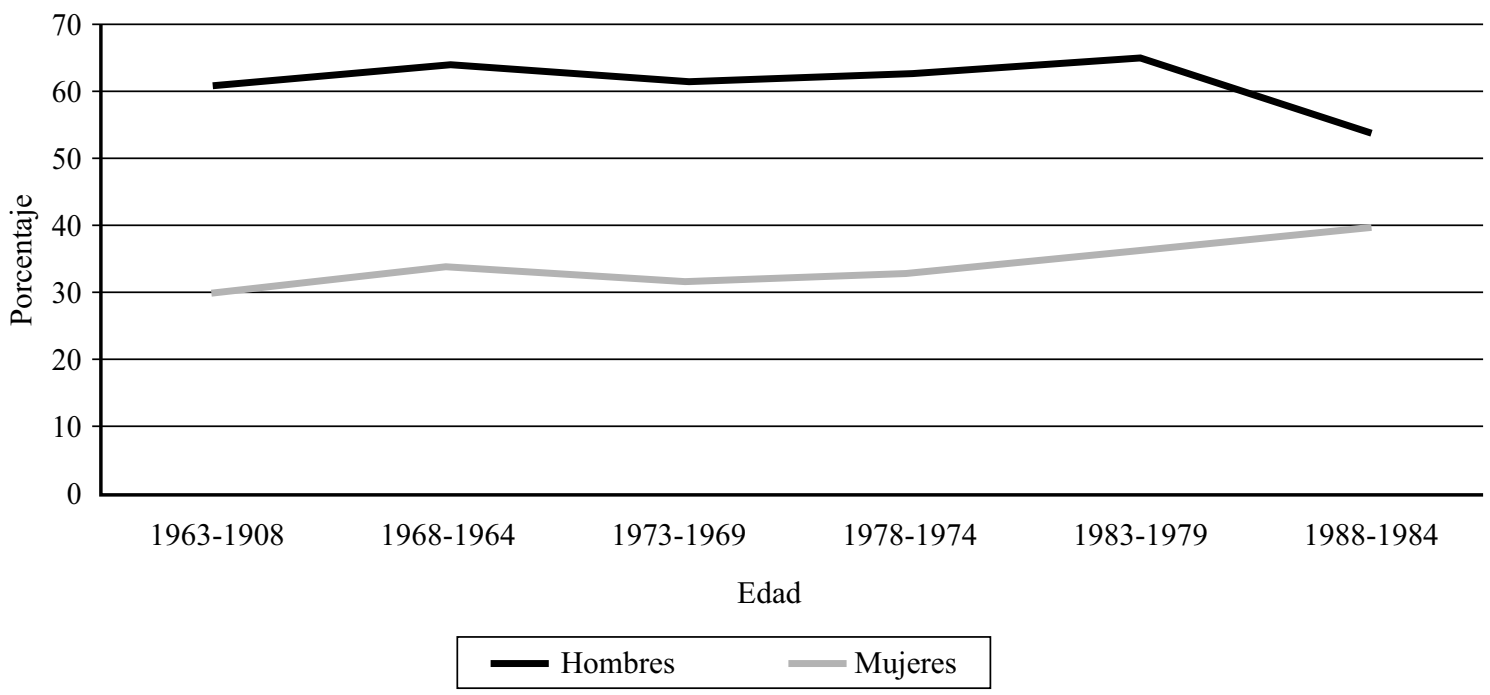

Fuente: procesamiento especial ENCV-2006

Figura 2. Chile. Porcentaje de iniciados/as sexualmente antes de los 18 años de edad según cohorte y género. 


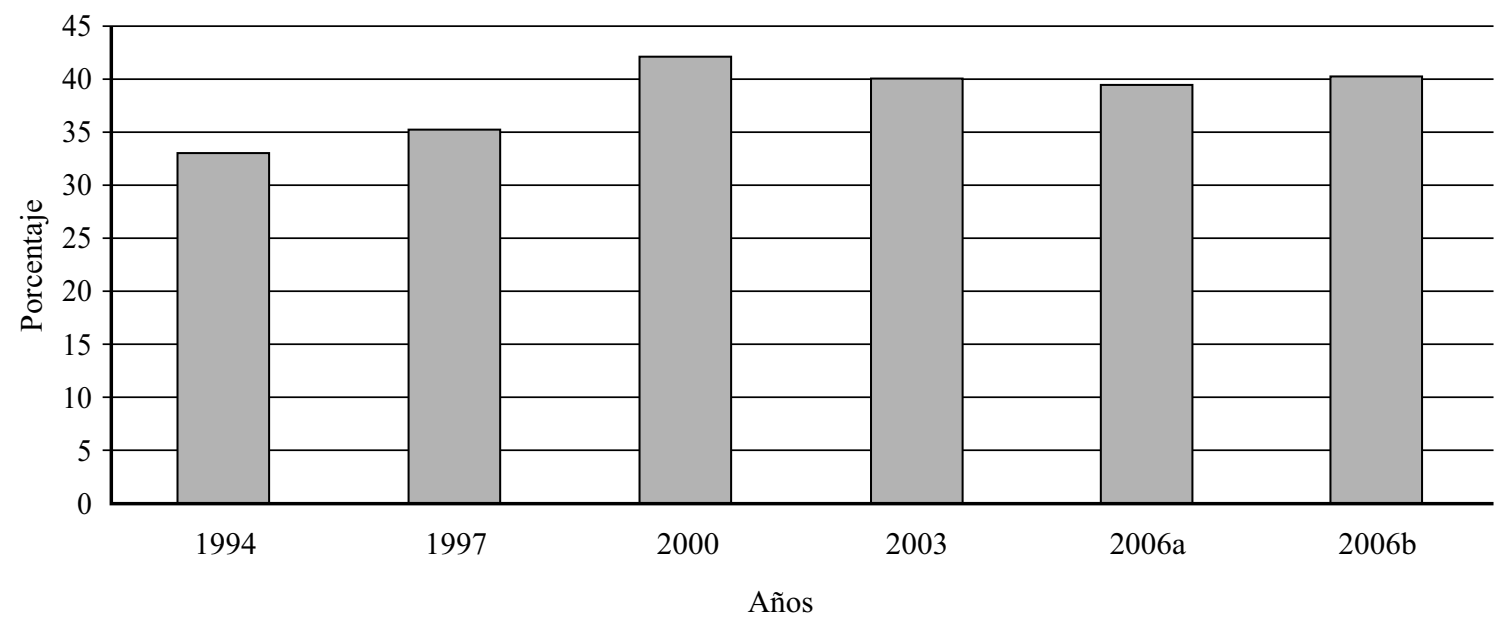

Fuente: 1994, 1997 y 2000: Informe Encuesta INJUV 2000, Gráfico sin número, p.28

(www,injuv,gob,cl/modules,php?name=Content\&pa=showpage\&pid=4); 2003: procesamiento especial base de microdatos de la IV Encuesta de Juventud del INJUV; 2006a: INJUV, 2006, Gráfico 96 (identifica solo a las RRSS penetrativas); 2006b: ENCV-2006, tabla III.3.3.1b. p. 48.

Figura 3. Chile. Porcentaje de adolescentes (hombres y mujeres) iniciados sexualmente, 1994-2006.

información. Y las ENJUVE, en particular sus publicaciones, no han indagado sistemáticamente en el tema. Con todo, el cuadro 123 de ENJUVE de 2006 (vínculo con la primera pareja sexual según género y tramo etario), sugiere que la gran mayoría se inicia antes de casarse, en el marco de esa peculiar institución chilena denominada "pololeo".

En síntesis, en línea con la experiencia de los países desarrollados, en Chile la iniciación sexual entre las mujeres se ha adelantado. Esto, sumado al casi seguro adelantamiento de la menarquia, ha elevado la probabilidad potencial de la maternidad adolescente en Chile (al menos hasta la década de 1990). Lamentablemente, las limitaciones de la información de base impiden estimaciones precisas de su impacto sobre la tendencia de la fecundidad adolescente. Ahora bien, su efecto real final sobre la fecundidad adolescente depende totalmente de la tendencia de las restantes variables intermedias, en particular el uso de anticonceptivos, mismo que se examina a continuación.

\section{Uso de anticonceptivos}

De acuerdo a todas las fuentes e indicadores, el uso de anticonceptivos en Chile ha aumentado en los últimos 15 años entre las y los adolescentes sexualmente activos. En efecto, el uso de anticoncepción en la primera relación sexual un indicador cada vez más usado por las limitaciones ya explicadas del uso actual ${ }^{11}$ - se elevó significativamente entre 2003 y 2006 (Figura 1). Este aumento puede deberse a las campañas contra el VIH/SIDA que promovieron el uso del preservativo. De hecho, al desagregar por método usado, el preservativo presenta la mayor alza por lejos ${ }^{\mathrm{e}}$. Con todo, aún se trata de una proporción baja y que en 2006 implicaba que más del $40 \%$ de las iniciaciones de adolescentes tenía riesgo de terminar en embarazo. Se trata de un indicador preocupante, porque -como ya se ha mostrado- la iniciación sexual crecientemente se desliga del matrimonio y de los propósitos reproductivos, por lo que la iniciación desprotegida puede implicar embarazos no deseados entre las adolescentes.

En la ENCV 2006 se hacen algunas consultas sobre uso actual de anticonceptivos que revelan, por ejemplo, que cerca del $60 \%$ de la población

(e) Según la ENCV 2006, el 50\% de la población de 15 a 19 años iniciada sexualmente, usó preservativo en su primera relación (Minsal. 2007. Tabla III.3.3.2b). 
adolescente sexualmente activa usa algún método para prevenir embarazo ${ }^{12}$, aunque no hay información para precisar la calidad y regularidad de este uso (y como ya se dijo parte de estas usuarias ya han sido madres). Así, un $40 \%$ de los y las adolescentes sexualmente activos tiene probabilidades altas de ser madre o padre. Como la actividad sexual durante la adolescencia se ha incrementado, este $40 \%$ representa una cantidad creciente de casos de población expuesta al riesgo de embarazo adolescente.

En síntesis, la iniciación sexual protegida se está extendiendo y puede ser uno de los factores clave tras el descenso de la fecundidad adolescente entre 1998 y 2004. Con todo, aunque presenta niveles comparativamente bajos (como se mostrará más adelante), aún se requiere más análisis para estimar cuánto de esta desprotección inicial se asocia específicamente a riesgo de embarazo no deseado.

\section{La desigualdad en materia de determinantes próximos}

El principal hallazgo es que la desigualdad social de la iniciación sexual presenta un patrón atenuado según la ENJUVE, 2006 o asistemático
Tabla 1. Chile. Uso de anticonceptivos en la primera relación sexual según edad, ambos sexos, 2003 y 2006.

\begin{tabular}{|c|c|c|c|}
\hline Años encuesta & $\mathbf{1 5 - 1 8}$ & $\mathbf{1 9 - 2 4}$ & $\mathbf{2 5 - 2 9}$ \\
\hline $\mathbf{2 0 0 3}$ & 41.3 & 37.5 & 28.3 \\
\hline $\mathbf{2 0 0 6}$ & 58.8 & 50.9 & 37.0 \\
\hline
\end{tabular}

Fuente: ENJUVE 2003 y 2006, procesadas con INJUMAP.

según la ENCV, 2006. Según la ENJUVE 2006, tanto para hombres como para mujeres, sólo el grupo más acomodado ( $\mathrm{ABC} 1,9 \%$ de $10 \mathrm{~s}$ jóvenes) presenta una notoria menor proporción de iniciadas/os sexualmente (Tabla 2), lo que contrasta con el gradiente sistemático en materia de maternidad adolescente ${ }^{13}$. Por su parte, la ENCV 2006 muestra, tanto para hombres como para mujeres, un gradiente clásico entre el quintil 1 (más pobre) y el quintil 4, pero el quintil más rico (quinto quintil) se aparta de la relación y registra niveles elevados de iniciación sexual (Tabla 2).

Dado que estos resultados, en particular el de la ENCV, pueden estar afectados por factores extrínsecos ${ }^{\mathrm{g}}$, se procedió a estimar regresiones logísticas para controlarlos (Tabla 3). El resultado para el caso de ENCV es claro: la edad, como se espera, aumenta la probabilidad

Tabla 2. Chile. Porcentaje de adolescentes iniciados sexualmente por sexo y según condición socioeconómica, según dos fuentes de 2006.

\begin{tabular}{|c|c|c|c|c|c|c|c|}
\hline \multirow{2}{*}{ Año } & \multirow{2}{*}{ Sexo } & Total & \multicolumn{5}{|c|}{ Estrato socioeconómico $^{\mathrm{f}}$} \\
\hline & & & Más alto & Alto & Medio & Medio-bajo $^{\text {Bajo }}$ \\
\hline \multirow{2}{*}{ ENJUVE 2006 } & Mujeres & 35.2 & 25.3 & 35.5 & 33.1 & 38.7 & 38.2 \\
& Hombres & 44.0 & 38.5 & 43.3 & 46.1 & 42.8 & 47.2 \\
\hline \multirow{2}{*}{ ENCV 2006 } & Mujeres & 34.1 & 37.9 & 14.1 & 25.3 & 42.1 & 50.4 \\
& Hombres & 45.5 & 46.1 & 38.4 & 34.8 & 53.9 & 59.6 \\
\hline
\end{tabular}

Fuente: procesamiento especial base de microdatos de la ENAJU 2006 y de la ENCV 2006.

(f) Las clasificaciones socioeconómicas No son comparables entre ambas encuestas, ya que la ENJUVE usa el procedimiento estándar de los estudios de mercado en Chile (metodología ESOMAR por lo cual el grupo socioeconómico más elevado es el denominado $\mathrm{ABC} 1$, que representa menos del 10\% de la población), mientras que la ENCV usa un índice compuesto que permite ordenar a todos los hogares y luego segmentar en quintiles, por lo que cada grupo representa, en principio, al $20 \%$ de los hogares.

(g) Por ejemplo la edad, ya que en una muestra pequeña las distribuciones por edad simple del grupo 15 a 19 pueden estar sesgadas, lo que genera efectos de composición (si hay una proporción exagerada de mujeres de 19 los niveles de fecundidad adolescente pueden ser altos porque estas tienen más tiempo de exposición al riesgo), que distorsionan los resultados y hacen riesgosas las comparaciones. 
Tabla 3. Chile. 2006. ENCV. Coeficientes de las razones de disparidad (Odds ratios) de las regresiones logísticas sobre la condición de actividad sexual y el uso de anticonceptivos en la primera relación sexual, población entre 15 y 19 años.

\begin{tabular}{|l|c|c|}
\hline Variable & \multicolumn{2}{|c|}{ Razón de disparidad (Odds ratio) } \\
\hline & $\begin{array}{c}\text { Estar } \\
\text { sexualmente } \\
\text { iniciado/a }\end{array}$ & $\begin{array}{c}\text { Haber usado } \\
\text { anticonceptivos } \\
\text { la primera } \\
\text { relación sexual }\end{array}$ \\
\hline Edad & 2.227 & .637 \\
\hline Zona (Urbana): & 1.029 & .916 \\
\hline Sexo (Hombre): & 2.062 & 1.149 \\
\hline $\begin{array}{l}\text { Quintil 1 } \\
\text { (más pobre) }\end{array}$ & 2.480 & .098 \\
\hline Quintil 2: & 1.695 & .123 \\
\hline Quintil 3: & .791 & .307 \\
\hline Quintil 4 & .707 & .189 \\
\hline Constante & 0.000 & 28.2 \\
\hline
\end{tabular}

Fuente: cálculos propios usando la base de microdatos de las encuesta ENCV 2006.

Nota: Todos los coeficientes significativos al $0.01 \%$

de estar iniciado/a sexualmente (simple efecto de exposición al riesgo, no de cambio conductual entre cohortes). Los adolescentes urbanos tienen unas chances (odds ratios) levemente superiores $(3 \%)$ a sus contrapartes rurales de haberse iniciado sexualmente. El género también hace una diferencia significativa y en el sentido esperado; en efecto, los hombres tienen una chance de estar iniciados sexualmente que es dos veces la de las mujeres, controlados los otros factores del modelo. Finalmente, y este era el objetivo del procedimiento multivariado, se confirma la anomalía observada en el análisis bivariado, pues luego de controlar por edad, sexo y zona de residencia, los/as adolescentes de los dos quintiles socioeconómicos inferiores tienen el doble o más chance de estar sexualmente iniciados/as que el quintil superior, pero los/as de los quintiles tres y cuatros tienen chances entre un 20 y un $30 \%$ menores que el quintil superior.
En síntesis, la desigualdad en materia de iniciación sexual presenta un patrón más complejo que en el pasado, con indicios de una tendencia hacia su disminución o al menos su irregularidad, lo que resta importancia a este determinante próximo como factor explicativo de la pertinaz desigualdad social en materia de maternidad adolescente.

Como contrapartida, en la misma Tabla se exponen los coeficientes de las relaciones de disparidad del indicador de uso de anticonceptivos más relevante según lo expuesto en este trabajo (uso en la primera relación), y los resultados muestran una desigualdad muy marcada y en el sentido predicho -que está en directa relación con la desigualdad en materia de reproducción adolescente-, a saber: a mayor nivel socioecónomico mayores índices de uso. En efecto, las chances de haber usado anticoncepción en la primera relación sexual son entre una décima (quintil más pobre) y un tercio (quintil 3) de las chances del quintil superior (Tabla 2).

\section{DICUSIÓN}

En los países actualmente desarrollados, el adelantamiento de la iniciación sexual (en particular entre las mujeres) no condujo a una elevación de la fecundidad adolescente ${ }^{\mathrm{h}}$. Más aún, lo que se ha verificado es una postergación generalizada de la iniciación reproductiva, lo que fue incluido como rasgo inherente del régimen demográfico "de modernidad avanzada" que describe la teoría de la segunda transición demográfica ${ }^{14}$. La razón es simple: generalización del uso de anticonceptivos desde la primera relación sexual. Y esto se ha basado en: i) deseo/decisión de los y las adolescentes, que parecen haber internalizado masivamente las adversidades que le significaría la procreación temprana; ii) una actuación facilitadora (o al menos no entorpecedora) de la familia para que los y las adolescentes ejerzan el control sobre

(h) Por cierto, hay diferencias entre países desarrollados, siendo sobresaliente el caso de Estados Unidos, cuya fecundidad adolescente es bastante más alta que el promedio de Europa Occidental y Japón. Para una interpretación de este fenómeno ver: Bongaarts y Cohen, 1998. 
su reproducción biológica; iii) políticas públicas integrales de información y educación en materia sexual y de aseguramiento de acceso a anticonceptivos (y en muchos países aborto) a los y las adolescentes que los requieran. Las encuestas Fertility and Family Survey (www.unece.org/pau/ffs/ffs.htm) levantadas en varios países desarrollados en la década de 1990, ya mostraban una prevalencia de uso de anticonceptivos en la primera relación sexual de $70 \%$ o más entre las mujeres ${ }^{\mathrm{i}}$.

En Chile la situación es bastante diferente, porque sólo hace unos pocos años se superó la barrera del $50 \%$ de iniciación sexual protegida entre adolescentes y las desigualdades sociales de esta cobertura son enormes y pertinaces. Por ello, ampliar este acceso parece central para cumplir con compromisos internacionales ${ }^{15} \mathrm{y}$ el objetivo sanitario definido por el Ministerio para el período 2000-2010 j. Lo anterior se refuerza por el hallazgo más relevante de este estudio: las desigualdades son mayores en uso de anticonceptivos que en edad de iniciación sexual. La experiencia de los países desarrollados sugiere que podría acentuarse en el futuro. Y esto es clave porque los cambios en estas dos variables intermedias tienen efectos poderosos sobre la trayectoria de la fecundidad adolescente (Ali y Cleland 2005).

Claro está que procesos o fenómenos socioeconómicos o culturales -por ejemplo: las tendencias del mercado de trabajo juvenil, el avance de la educación media y superior, las transformaciones de las relaciones de poder dentro de la familia y entre hombres y mujeres, la expansión de los medios de comunicación de masas, la expansión de los denominados "valores post-industriales", etc.- inciden en estas dos variables. Pero lo hacen de una manera compleja, en alguna medida contigente y en plazos largos ${ }^{16}$. Considerando la urgencia que imponen los indicadores de nivel y de desigualdad, no hay espacio para plazos largos, por lo que las políticas públicas deben actuar de manera directa $\mathrm{y}$ decidida sobre las variables intermedias. Y los hallazgos de este trabajo subrayan la prioridad de universalizar el acceso a medios anticonceptivos desde la primera relación sexual, para que cada adolescente pueda decidir libre, informada y conscientemente sobre su uso, sin restricciones adicionales a las establecidas legalmente.

En el plano de la investigación futura este estudio ratifica la urgente necesidad de datos y análisis especializados (sociodemográfico) sobre este tema, ya que la información disponible en materia de determinantes próximos (en particular conducta sexual y anticonceptiva) proviene de fuentes no especializadas y es bastante limitada.

\section{REFERENCIAS}

1. BOZON M. À quel âge les femmes et les hommes commencent-ils leur vie sexuelle? Comparaisons mondiales et évolutions récentes. Population et sociétés Junio 2003: 391: 1-4.

2. Ibidem

3. RODRÍGUEZ J. Reproducción adolescente y desigualdades en América Latina y el Caribe: un llamado a la reflexión y a la acción. Madrid OIJCEPAL-UNFPA 2009.

4. Ibidem.

5. RODRÍGUEZ, J. 2009, op.cit; RODRÍGUEZ J. Reproducción en la adolescencia: el caso de Chile y sus implicaciones de política. Revista de la CEPAL: 2005: 86: 123-146; DIDES C. BENAVENTE C. Diagnóstico de la situación del embarazo en la adolescencia en Chile. FLACSO 2008; MOLINA R, MOLINA T, GONZÁLEZ E. Salud sexual y reproductiva en la adolescencia. Mediterráneo Chile 2003.

6. RODRIGUEZ J. 2009 op.cit; ALI M, CLELAND J. Sexual and reproductive behaviour among single women aged 15-24 in eight Latin American countries: a comparative analysis Social Science \& Medicine 2005 60(6): 1175-1185; BREIBAUER C. MADDALENO M. Youth: choices and change. Promoting healthy behaviors in adolescents Washington, PAHO 2005; MOLINA R. MOLINA T. GONZẢLEZ, E. 2003, op.cit.; BONGAARTS J. COHEN B. Adolescent reproductive behavior in the developing World. Studies in Family Planning 1998: 29(2); Naciones Unidas. Programa de Acción de la Conferencia Internacional sobre la Población y el

(i) Por ejemplo: Suiza: $85 \% .5$ cohorte nacida en 1970-1974 e Italia: 75\% cohorte nacida entre 1971 y 1975 . Tablas estándar disponibles en www.unece.org/pau/ffs/ffs.htm.

(j) $30 \%$ de reducción de los embarazos adolescentes no deseados (Minsal (2002). Objetivos sanitarios para la década 2000-2010, El Vigía, 5(15), Minsal. Santiago. Edición especial, http://epi,minsal,cl/epi/html/elvigia/vigia15.pdf). 
Desarrollo. Nueva York 1994 [en línea] www,unfpa,org/icpd/icpd-programme_spa,cfm\#ch7.

7. CONASIDA Estudio Nacional de Comportamiento Sexual, Primeros Análisis, Chile 2000. Santiago de Chile Ministerio de Salud 2000.

8. ALI M, CLELAND J. 2005: op.cit.

9. BONGAARTS, J. A framework for analyzing the proximate determinants of fertility, Population and Development Review: 1978: 4: 1.

10. RODRIGUEZ, 2009: op.cit.

11. RODRIGUEZ 2009: op.cit.; DI CESARE M, RODRÍGUEZ J. Análisis micro de los determinantes de la fecundidad adolescente en Brasil y Colombia. Papeles de Población: 2006: 48: 107-140

12. MINSAL. Encuesta Nacional de Calidad de Vida, 2006, MINSAL Santiago 2007: Tabla III.3.3.3b
13. DIDES C. BENAVENTE C. 2008: op. cit.; RODRÍGUEZ J. 2005: op.cit.

14. ALVES J. CAVENAGHI S. Timing of childbearing in below replacement fertility regimes: how and why Brazil is different? Documento presentado en la Sesión 28 "Timing of childbearing" de la XXVI Conferencia Internacional de Población de la IUSSP Marrakech Marruecos 27 de septiembre a 2 de octubre de 2009.

15. NACIONES UNIDAS, 1994: op.cit.

16. RODRÍGUEZ, J. 2009: op. cit.; BONGAARTS J. COHEN B. 1998: op.cit.

Recepción: 21 de diciembre de 2009 Aprobación: 10 de marzo de 2010

Usted puede comentar éste y otros artículos publicados en la Revista Chilena de Salud Pública, enviando un correo electrónico a revistasp@med.uchile.cl 